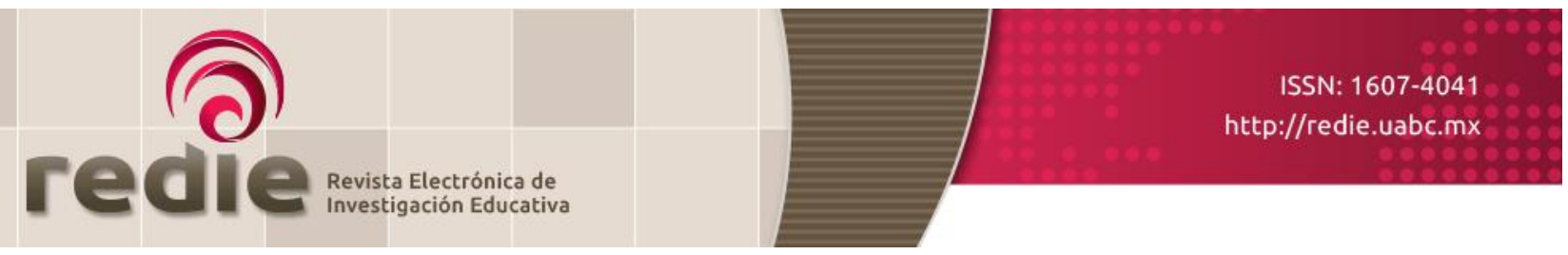

Vol. 20, Núm. 2, 2018

\title{
ConVivim: aplicación de un programa para aprender a convivir democráticamente
}

\section{ConVivim: Application of a Program to Learn to Coexist Democratically}

\author{
Roser Grau Vidal (*) roser.grau@uv.es \\ Laura García Raga (*) laura.garcia@uv.es \\ (*) Universidad de Valencia \\ (Recibido: 4 de octubre de 2016; Aceptado para su publicación: 22 de diciembre de 2016)
}

Cómo citar: Grau, R. y García, L. (2018). ConVivim: aplicación de un programa para aprender a convivir democráticamente. Revista Electrónica de Investigación Educativa, 20(2), 80-92. https://doi.org/10.24320/redie.2018.20.2.1619

\section{Resumen}

En las últimas décadas, la convivencia se ha convertido en uno de los desafíos más importantes de las sociedades del siglo XXI, y su versión educativa en un taller práctico de construcción de una ciudadanía comprometida con los valores cívicos de la democracia. En este contexto toman valor las estrategias enmarcadas en el modelo de "ayuda entre iguales", como el programa ConVivim, cuyo diseño e implementación se presenta en este trabajo con el objetivo de demostrar su impacto en la mejora de la convivencia democrática. El escenario de investigación es una escuela ubicada en un barrio en situación de vulnerabilidad social, donde se aplica un diseño cuasi-experimental, pretest-postest, a 83 miembros de su comunidad educativa. Los resultados muestran el éxito de la intervención, transferible a otras realidades internacionales, aun cuando resulta evidente la exigencia de seguir trabajando para que la escuela se convierta en una institución referente para la cohesión social.

Palabras clave: Escuela, mediación, relación entre pares, resolución de conflictos.

\begin{abstract}
In recent decades, coexistence has become of the most significant challenges of $21^{\text {st }}$ century society, and in the field of education, this has become a hands-on workshop to shape citizens who are committed to the civic values of democracy. It is within this context that strategies included in the "help between peers" model, such as the ConVivim program, gain importance. This study presents the design and implementation of ConVivim in order to prove its impact in improving democratic coexistence. The setting for the study is a school located in a vulnerable neighborhood, and a quasi-experimental, pretestposttest design was used with 83 members of the educational community. The results show the success of the intervention, which can be transferred to other international contexts, despite clear evidence of the need for further work to make schools a model institution for social cohesion.
\end{abstract}




\section{Introducción}

Aprender a convivir sigue siendo uno de los grandes retos del siglo XXI (UNESCO, 2015), en una sociedad donde la violencia aparece con excesiva fuerza: guerras y conflictos armados continúan asolando la realidad de cada día en muchos países; el terrorismo golpea buena parte del planeta, la violencia de género sigue presente y los casos de acoso escolar y laboral son realmente preocupantes. Parece que la tolerancia a la violencia aumenta (más la que vemos televisada), insensibilizándonos ante ella (Yubero, Larrañaga y Morales, 2003). Son síntomas de una sociedad, que sigue sin saber cómo resolver el camino hacia la paz. La educación, como afirmó Nelson Mandela, "es el arma más poderosa que puedes usar para cambiar el mundo", por ello la escuela es el lugar idóneo para potenciar la transformación de la cultura violenta en una cultura pacífica (Tuvilla, 2004).

Educar para la paz implica el aprendizaje de la convivencia, que sólo puede alcanzarse educando pacíficamente, incluyendo en la vida escolar los presupuestos de la cultura de paz (Boqué, Pañellas, Alguacil y García, 2014); aprender a gestionar la toma de decisiones, actuar ante las injusticias, pensar en los demás y en el beneficio común, alimentar la participación directa adquiriendo responsabilidades desde el inicio de la escolarización, son aspectos a tener presentes en el mundo escolar.

Una de las formas de mejorar la convivencia (probablemente la más eficaz) es aumentar y enriquecer la participación. Lo que se considera propio se defiende y se respeta; la norma que es consensuada se cumple y se valora. Ante la imposición es fácil responder con la indiferencia o con la rebeldía; nos referimos a una participación auténtica, profunda y, en definitiva, educativa (Santos-Guerra, 2003, p. 108).

Es cierto que educar a la sociedad actual es una utopía, y empezar a cambiar la mirada para educar a la sociedad del futuro es un reto difícil, aun cuando no deja de ser asumible. Numerosos investigadores alrededor del mundo siguen preocupándose por las dificultades derivadas de las relaciones interpersonales, trabajando con el objetivo de encontrar "soluciones" que ayuden a promover iniciativas que fomenten la ayuda, la empatía, la tolerancia, la igualdad, la dignidad y la cooperación. La escuela es, por tanto, un lugar desde el cual partir hacia la construcción de una sociedad más horizontal y democrática, a través de herramientas que aboguen principalmente por la convivencia pacífica y libre de estereotipos (Puig y Morales 2015; Harber y Sakade 2009).

Los profesionales de la educación demandan constantemente iniciativas y formación que les ayuden a enfrentarse a una realidad cambiante, en la medida de que los conflictos y la violencia escolar son temáticas que siguen preocupando al profesorado, a investigadores y a las propias administraciones educativas (Gómez y Gaymard, 2014); las urgencias de trabajar conjuntamente con las familias y el alumnado, construyendo una relación horizontal, mediante la reflexión compartida, la investigación y la toma de decisiones es un reto inexcusable para el futuro inmediato (Santos-Guerra, 2009).

Los centros educativos siguen siendo lugares donde estudiantes y profesorado pasan largas horas construyendo microsociedades en las que habitualmente se desarrollan acontecimientos, se viven experiencias y, en definitiva, se convive con el entorno; por tanto, la escuela no deja de ser un espacio de relación y aprendizaje entre iguales y adultos, y eso implica afrontar situaciones de dificultad que deben gestionarse positivamente (Viguer y Avià, 2009). Si analizamos desde una perspectiva más global el significado de convivencia, resulta sorprendente la carga de contenido que aglutina el concepto: convivir, además de respetar, tolerar y valorar, es aprender a sentir, sonreír, amar y disfrutar, lo que otorga al papel de los otros un sentido fundamental, garantizando el ser felices siempre que los demás también lo sean. Uno de los objetivos del aprendizaje de la convivencia, según Funes (2000), debe ser el sentirse capaz de "convivir con la diferencia, sin que ello sea irreconciliable, traumático o fuente de malestar" ( $p$. 100). En consecuencia, el papel de las escuelas es imprescindible, ya que deben contribuir al aprendizaje que supone el establecer relaciones interpersonales sanas y de calidad, apostando por principios de igualdad, preocupación, tolerancia y empatía con los demás (Schulman, 2002).

Si nos centramos en el ámbito que nos atañe, cabe destacar que aprender a relacionarse implica también detectar situaciones de dificultad por las que las personas que nos rodean transitan cada día, con la finalidad de ser capaces de prestar ayuda si la necesitan, aun asumiendo el reto que implica el aprender a 
ayudar y a pedir ayuda. Resulta imprescindible la "revitalización de la ayuda en la vida cotidiana, potenciando el encuentro y la colaboración con el otro (Caba-Collado y López-Atxurra, 2013, p. 123). Cuando nos referimos al concepto de ayuda, hablamos de la necesidad de cuidarnos, buscando la manera de crear un clima de convivencia pacífico, motivador, enriquecedor, alegre y, en definitiva, feliz: cuidarse implica preocuparse por los demás, desplazando el individualismo y el egoísmo por el sentimiento de comunidad y de grupo: "Las escuelas pueden, y deberían, jugar un papel importante en el desafío del maltrato entre iguales, creando una atmosfera de cuidado, es decir, una atmósfera donde los alumnos son motivados a ayudar a otros" (Cowie y Fernández, 2006 p. 293).

Cabe destacar que son muchas las opciones que expertos en la temática proponen, encaminadas a promover la puesta en marcha de iniciativas cuyos objetivos son el aprendizaje de la convivencia y, por ende, la práctica de la ayuda entre iguales. Poner en funcionamiento este tipo de programas proporciona la oportunidad para que los niños se pongan en el lugar de los demás, aprendiendo a escuchar sus necesidades y promoviendo un ambiente de conciliación y respeto (Fernández, 2008).

Sin duda, la ayuda entre iguales potencia que sea el alumnado quien asuma la responsabilidad de atender las necesidades que la comunidad educativa demanda, especialmente las de sus compañeros. Deben ser ellos quienes impulsen acciones encaminadas al desarrollo social, personal y comunitario de la escuela, con el apoyo incondicional del profesorado y de los agentes externos (asociaciones, entidades, etc.), que formen parte activa del centro. La ayuda entre iguales es un poderoso mecanismo de prevención y resolución de conflictos, dado que otorga protagonismo al propio alumnado, testigo de excepción de los distintos tipos de violencia escolar (Torrego, 2013, p. 10).

Numerosos estudios realizados a nivel internacional demuestran que en los centros educativos que han decidido poner en funcionamiento iniciativas de ayuda entre iguales, los niños desarrollan actitudes positivas y de apoyo hacia las víctimas (Cowie y Fernández, 2006), lo que convierte a este tipo de programas en herramientas de especial valor en contextos en situación de vulnerabilidad social. Según Cowie y Wallace (2000), los principales motivos por los que los programas de ayuda entre iguales pueden funcionar adecuadamente son los siguientes:

- Los iguales detectan la violencia en etapas más tempranas que los adultos.

- Es más probable que los jóvenes confíen en algún compañero que en un adulto.

- Las víctimas sienten que tienen a alguien a quien acudir para solucionar el problema.

- A los profesores a menudo les falta tiempo y recursos para ocuparse de todos los problemas interpersonales que les llegan a lo largo de un día.

- Los ayudantes adquieren habilidades interpersonales y aprenden sobre ciudadanía activa.

- La escuela es percibida por la comunidad educativa como una organización que cuida.

- El alumnado ayudante en ocasiones ha sido víctimas de violencia y, por tanto, ha desarrollado la empatía por la experiencia y esto le permite su inclusión en un grupo de iguales en el que predomina el apoyo y la ayuda.

Es evidente que la apuesta por la implementación de programas de ayuda entre iguales es un reto que marca la diferencia, ya que quienes consideran a los alumnos capaces de afrontar situaciones de dificultad recurriendo a sus propios compañeros, están dando una oportunidad a la construcción de una sociedad más democrática e igualitaria. Por ello, aprender a ayudar al otro deberá ser un objetivo más en el camino hacia una educación de calidad y, por extensión, hacia un mundo más justo y feliz.

\section{Método}

La investigación que presentamos tiene un diseño cuasi-experimental pretest-postest. El proceso consiste en la realización de una evaluación inicial, aplicación de un programa y evaluación del efecto del mismo. Es importante mencionar que los cuestionarios se han administrado de manera aleatoria entre el alumnado y el profesorado participante en ambos momentos de la evaluación para evitar efectos adversos. 
Se contó con un total de 83 participantes divididos en dos grupos de estudio, 67 son alumnos de 20. ciclo de educación infantil (4-6 años) y educación primaria (6-12 años), y 16 pertenecen al claustro de profesorado del centro. En cuanto a las características socio-demográficas, en el primer grupo 49\% son chicas y $51 \%$ chicos, de edades comprendidas entre los 5 y los 12 años. Han formado parte de la investigación el alumnado del 20. ciclo de educación infantil 4 años (12\%) e infantil 5 años (16\%), así como de los seis cursos de educación primaria: $9 \%$ de primero, 12\% de segundo, $9 \%$ de tercero, 15\% de cuarto, $11 \%$ de quinto y $16 \%$ de sexto. Respecto al profesorado, $75 \%$ son mujeres y $25 \%$ hombres, cuya experiencia varía entre los 2 y los 44 años de trayectoria docente (tabla I).

Tabla I. Características del profesorado participante

\begin{tabular}{|c|c|c|c|c|c|c|c|}
\hline Sexo & & $\begin{array}{l}\text { ¿Primer } \\
\text { año en el } \\
\text { centro? }\end{array}$ & & ¿Tutor? & & $\begin{array}{l}\text { Experiencia } \\
\text { (años) }\end{array}$ & \\
\hline \multirow[t]{2}{*}{ Hombre } & 4 & No & $77 \%$ & No & $50 \%$ & $0-5$ & 3 \\
\hline & & & & & & $6-10$ & 2 \\
\hline \multirow[t]{3}{*}{ Mujer } & 12 & Sí & $23 \%$ & Sí & $50 \%$ & $11-15$ & 4 \\
\hline & & & & & & $16-20$ & 2 \\
\hline & & & & & & +20 & 4 \\
\hline
\end{tabular}

El centro en el que se ha realizado la investigación está situado en un municipio cercano a la ciudad de Valencia (España), cuyas características lo han llevado a configurarse como Barrio de Acción Preferente (Decreto 157/1988). Es un núcleo urbano alejado del municipio al cual pertenece y, desde su nacimiento, se ha caracterizado por el narcotráfico, la marginalidad y la delincuencia. Este barrio, de difícil acceso, acechado por la violencia y la pobreza, se ha convertido en un gueto, por lo que podemos afirmar que la casi totalidad de las familias que residen allí viven en situación de vulnerabilidad social.

La escuela está situada en el centro del barrio, categorizado como Centro de Acción Educativa Singular. Según la Orden del 4 de julio de 2001 de la Consejería de Educación de Valencia, "los centros docentes ubicados en un Barrio de Acción Preferente (BAP) o que escolaricen alumnado con necesidades de compensación educativa en un porcentaje igual o superior al 30\% del total del centro, se considerarán Centros de Acción Educativa Singular (CAES)". De acuerdo con esta normativa, cabe destacar que este tipo de centros educativos deben realizar las adaptaciones curriculares correspondientes, facilitando así la adquisición de las competencias básicas y dando respuesta a las necesidades sociales y educativas del alumnado del centro.

Por otra parte, respecto al proceso de recogida de información en ambos momentos de la evaluación, se ha utilizado un cuestionario elaborado por Ortega y Del Rey (2003), desarrollado luego a nivel internacional (Cangas, Gázquez, Pérez-Fuentes, Padilla y Miras, 2007; Gázquez, Cangas, Pérez y Lucas, 2009). Ambos constan de 12 ítems, 8 cerrados y 4 abiertos. Con la finalidad de adaptar el nivel de dificultad del instrumento para educación infantil y primer ciclo de educación primaria, han resultado 13 ítems cerrados. Esta adaptación consistió principalmente en la redacción de un discurso más sencillo y en convertir las opciones de respuesta en tres iconos, para facilitar su desarrollo. El cuestionario original se aplicó al alumnado de 2o. y 3er. ciclo y al profesorado, adaptando únicamente las escalas de respuesta.

Por otra parte, ConVivim es el programa de intervención socioeducativa aplicado, creado por el grupo de profesores que realizó la presente investigación. Hay que recordar que fue evaluado en un estudio piloto, a través de la implementación en un centro con características similares, en el que participaron 297 estudiantes y 54 docentes -grupo experimental y grupo control-, obteniendo resultados satisfactorios y proponiendo mejoras que que se presentan en este estudio (Grau, García-Raga y López-Martín, 2016). 
Se trata de un programa de mejora de la convivencia compuesto por dos grandes bloques, el primero formado por 4 estrategias que forman parte del ya mencionado modelo "Ayuda entre iguales" y el segundo por acciones de "Mediación artística". Ambos requieren formación previa del profesorado, ya que son ellos los responsables de implementar de forma directa todas las estrategias del programa, con la colaboración de los artistas en el caso de la mediación artística.

En cuanto al primero de los bloques, en concreto se ha trabajado la asamblea de aula (Freinet, 1971), el Pair reading (apadrinamiento lector) (Durán y Blanch, 2015; Valdebenito y Durán, 2014), el ocio alternativo al tiempo de patio (Volk, Dane, Marini y Vaillancourt, 2015) y el programa de alumnos ayudantes (Torrego, 2012). Se ha considerado seleccionar estas estrategias por ser las más idóneas en el contexto en el que se sitúa la escuela objeto de investigación. Además, de forma paralela se ha desarrollado un proyecto transversal de mediación artística (Moreno, 2010; Rodrigo-Montero, 2015), con la colaboración de la onc Amnistía Internacional, sección Comunidad Valenciana. Se consideró que sería muy motivador utilizar el arte como recurso de cohesión social e implicación del alumnado junto con estrategias más específicamente dirigidas a la mejora de las relaciones interpersonales. En la figura 1 se sintetiza gráficamente el programa:

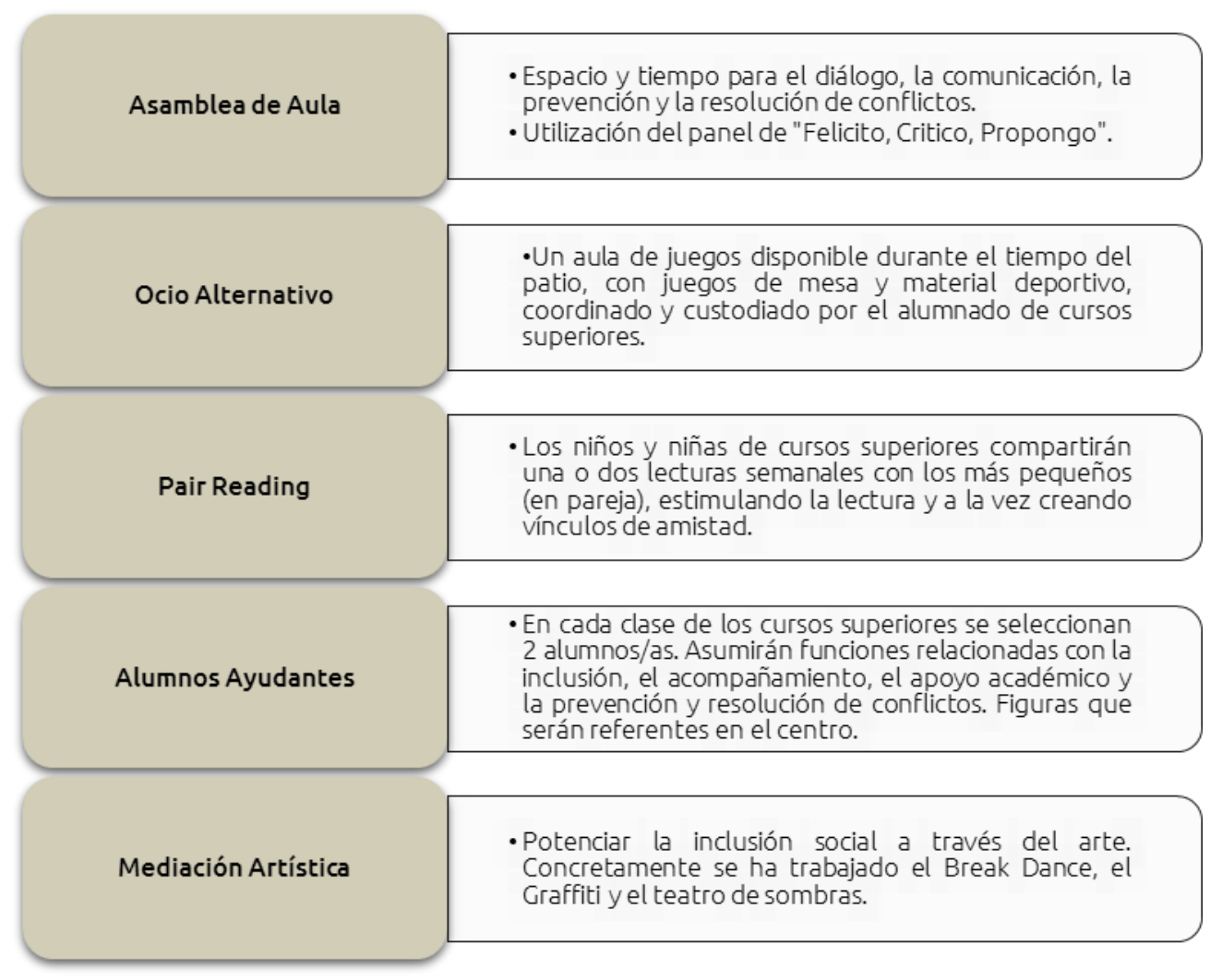

Figura 1. Programa ConVivim

Una vez aplicado y evaluado el programa, los datos obtenidos tanto en el pretest como en el postest se han procesado y analizado mediante el software spSS versión 22. Se ha realizado la prueba no paramétrica Wilcoxon para dos muestras relacionadas (pretest y postest) y la t de Student para comparar medias y comprobar diferencias estadísticamente significativas entre los diferentes momentos de evaluación para cada grupo de estudio. 


\section{Resultados}

Con la finalidad de presentar los resultados obtenidos de una forma ordenada se exponen por grupos de cursos, atendiendo a las adaptaciones de los instrumentos antes mencionadas.

Tabla II. Estadísticos descriptivos del segundo ciclo de Educación Infantil y primer ciclo de Educación Primaria

\begin{tabular}{l|cc|cc}
\hline Descriptivos & \multicolumn{2}{|c|}{ Pretest } & \multicolumn{2}{c}{ Postest } \\
\hline ítems & Media & $\begin{array}{c}\text { Desv. } \\
\text { típ }\end{array}$ & Media & Desv. \\
típ
\end{tabular}

En primer lugar, respecto al segundo ciclo de educación infantil y primer ciclo de educación primaria, podemos observar que los resultados han mejorado respecto a la evaluación inicial (tabla II). Cabe destacar un incremento de la mejora en los ítems 8, 13 y 14, ya que la media obtenida en el postest prácticamente llega a los 3 puntos. La media global después de la aplicación del programa es de 2.6 puntos, lo cual indica que las mejoras alcanzadas son destacables.

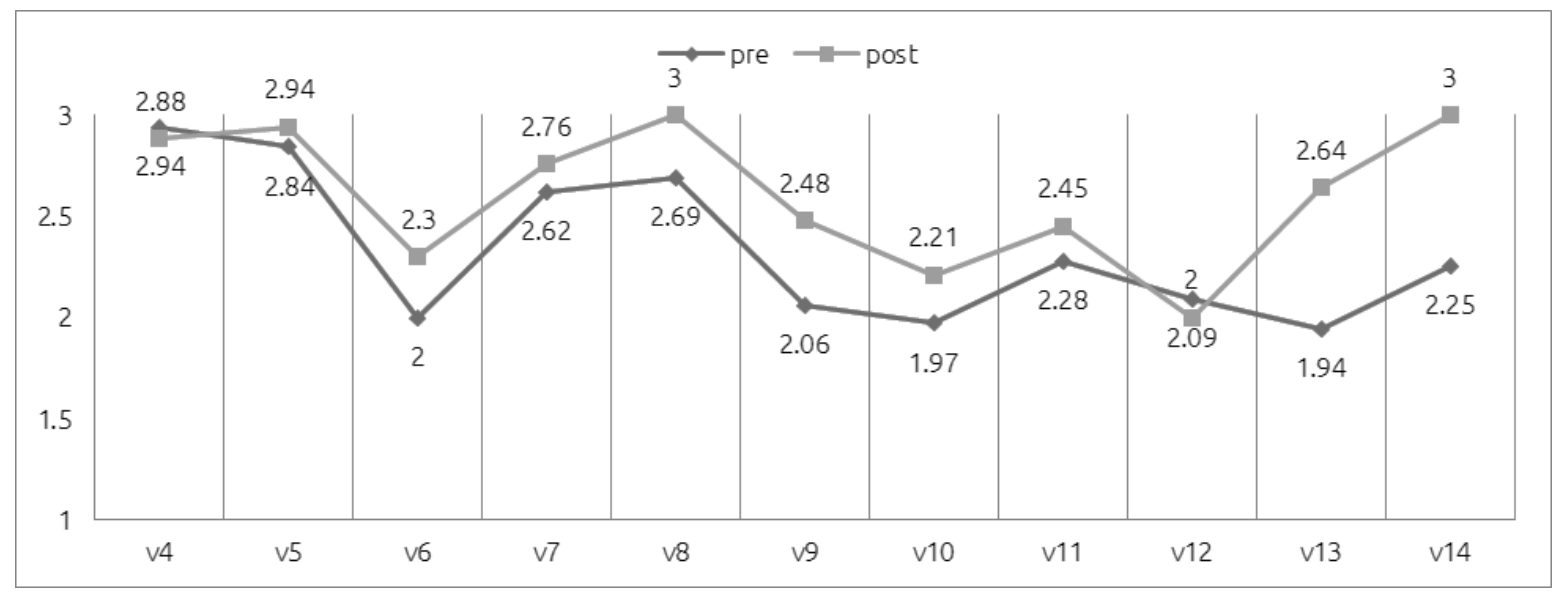

Figura 2. Comparación de medias obtenidas en pretest y postest en el segundo ciclo de Educación Infantil y primer ciclo de Educación Primaria

Por otra parte, dos de los ítems del postest han bajado ligeramente la media respecto al pretest, obteniendo un resultado prácticamente igual. El primero de ellos es el que hace referencia a la relación con los compañeros (ítem 4), lo cual puede deberse a la baja autoestima y autoconcepto de los menores. Es importante mencionar que el contexto en el que se encuentran inmersos no potencia el valor por la educación y la formación como futuros ciudadanos. Sin duda, este conjunto de variables influyen en el desarrollo personal y social del alumnado del centro y se ve reflejado en los resultados obtenidos. 
Por otra parte, cabe destacar que si analizamos los resultados del segundo y tercer ciclo de educación primaria, es fundamental resaltar la mejora que apreciamos en los datos obtenidos después del programa (tabla III y figura 3).

Tabla III. Estadísticos descriptivos correspondientes al segundo y tercer ciclo de primaria

\begin{tabular}{|c|c|c|c|c|}
\hline \multirow{2}{*}{$\begin{array}{l}\text { Descriptivos } \\
\text { Ítems }\end{array}$} & \multicolumn{2}{|c|}{ Pretest } & \multicolumn{2}{|c|}{ Postest } \\
\hline & Media & $\begin{array}{c}\text { Desv. } \\
\text { típ }\end{array}$ & Media & $\begin{array}{c}\text { Desv. } \\
\text { típ }\end{array}$ \\
\hline 4. ¿Cómo te llevas con tus compañeros en relación a principio de curso? & 1.64 & .742 & 1.59 & .701 \\
\hline 5. ¿Y con los profesores y las profesoras? & 1.39 & .659 & 1.41 & .657 \\
\hline 6. ¿Qué opinión crees que tienen de ti tus compañeros y tus compañeras? & 1.79 & .740 & 1.76 & .781 \\
\hline 7. ¿Qué opinión crees que tienen de ti tus profesores y tus profesoras? & 1.42 & .663 & 1.53 & .662 \\
\hline 8. ¿Hay diferencias en las normas de clase de unos profesores/as y de otros/as? & 1.27 & .452 & 1.79 & .592 \\
\hline 9. ¿Participan ahora más las familias en la vida del colegio? & 2.36 & .783 & 2.12 & .769 \\
\hline 10.1. Las familias no participan en nada. & 1.00 & .000 & 1.32 & .475 \\
\hline 10.2. ¿Participan las familias en las fiestas? & 1.24 & .435 & 1.68 & .475 \\
\hline 10.3. ¿Las familias van al colegio a recoger las notas? & 1.88 & .331 & 1.65 & .485 \\
\hline 10.4. ¿Las familias van al colegio si los llaman? & 1.42 & .502 & 1.59 & .500 \\
\hline 10.5. ¿Las familias van al colegio si su hijo va mal? & 1.79 & .415 & 1.82 & .387 \\
\hline 10.6. ¿Las familias forman parte del AMPA? & 2.00 & .000 & 1.94 & .239 \\
\hline 11.1. Enfrentamientos entre los grupos de alumnos/as y el/a profesor/a & 2.58 & 1.091 & 2.35 & .849 \\
\hline 11.2. Malas palabras en clase. & 1.79 & .820 & 2.06 & .547 \\
\hline 11.3. No se respetan las normas. & 1.67 & .692 & 2.24 & 699 \\
\hline 11.4. Los/as alumnos/as se insultan. & 1.30 & .529 & 2.09 & .668 \\
\hline 11.5. Los/as alumnos/as se pelean. & 1.27 & .626 & 2.12 & .537 \\
\hline 11.6. Hay grupitos que no se llevan bien. & 1.61 & .704 & 2.71 & .676 \\
\hline 11.7. Hay niños/as que no están integrados/as y se sienten solos/as. & 1.82 & .727 & 3.56 & .705 \\
\hline 11.8. Los/as profesores/as van cada uno a lo suyo. & 2.76 & .867 & 3.76 & .554 \\
\hline 11.9. Los/as alumnos/as piensan que los/as profesores/as no les entienden. & 2.45 & .711 & 3.38 & .739 \\
\hline 11.10. Los/as alumnos/as están desmotivados/as, se aburren. & 2.27 & .839 & 2.82 & .869 \\
\hline Total & 1.76 & .229 & 2.14 & .164 \\
\hline
\end{tabular}

Sin duda, prácticamente todos los ítems han evolucionado positivamente, pero es imprescindible hacer especial mención a las variables $11.4,11.5,11.6,11.7,11.8,11.9$ y 11.10, cuyos resultados superan la media esperada al inicio del estudio. Estos indicadores hacen referencia a comportamientos íntimamente relacionados con la convivencia escolar, como pueden ser: insultos, peleas, exclusión de niños de grupos, actitudes del profesorado y enfrentamientos entre grupos de alumnado, entre otros.

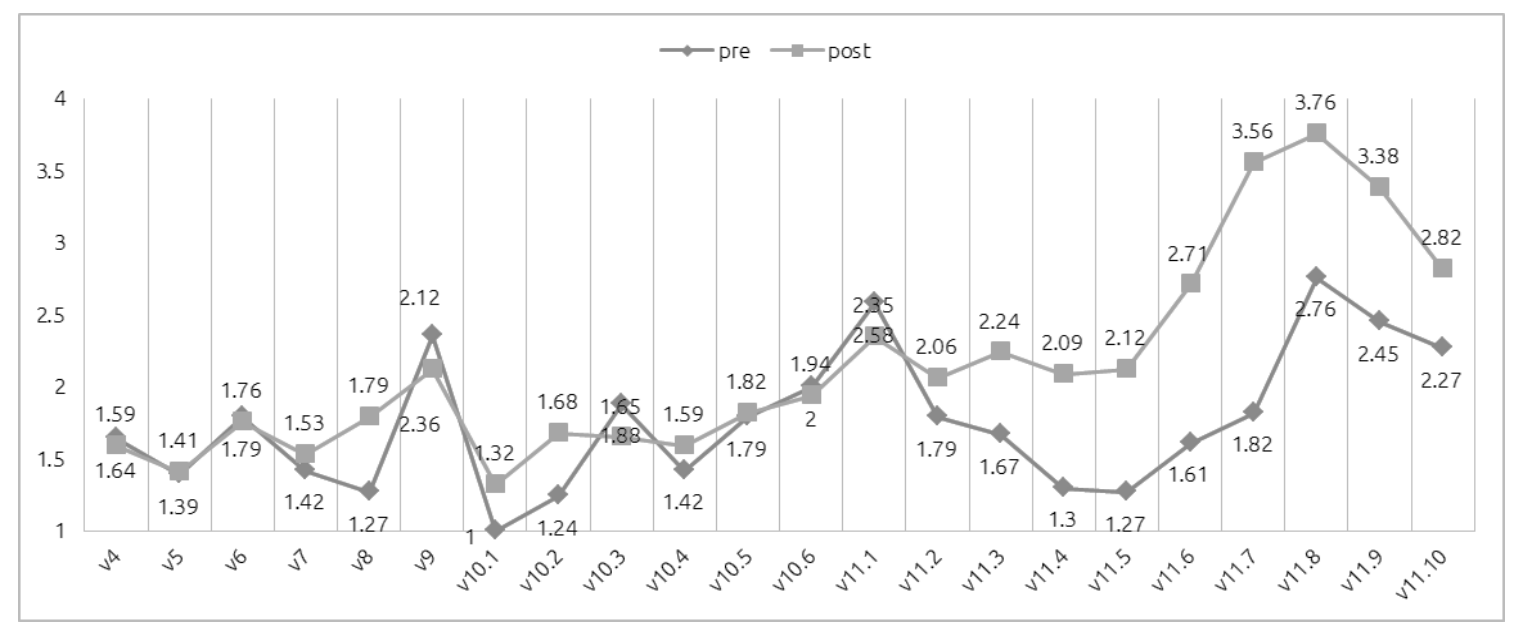

Figura 3. Comparación de medias obtenidas en pretest y postest en el segundo y tercer ciclo de Educación Primaria 
Es cierto que no todos los ítems han evolucionado positivamente, aunque son sólo dos los que no han experimentado cambios significativos. Es importante destacar el ítem 9, referente a la percepción del alumnado respecto a la actitud del profesorado, que obtuvo una media de 2.12 en el postest. Este resultado puede deberse a la falta de conexión y entendimiento entre el alumnado y el profesorado, lo que indica la necesidad de abordar esta dificultad en investigaciones posteriores. Respecto al ítem 10.3 (¿van las familias a recoger las notas al colegio?) resulta clave considerar la necesidad de potenciar la participación de las familias en el centro, por ello en el apartado siguiente se proponen opciones para trabajar en futuras líneas de investigación.

Por último, cabe señalar que el ítem 11.1 (enfrentamientos entre el alumnado) es una cuestión en la que ya se está trabajando, aunque debemos ser conscientes de que no todos los resultados se viven y se experimentan durante el primer año de funcionamiento, por lo que se debe apostar por el seguimiento de las estrategias del programa.

El último grupo de estudio corresponde al profesorado del centro. Los resultados demuestran que está presente tanto la motivación como la exigencia, sin dejar paso a la desmotivación y a la pérdida de interés.

Tabla IV. Estadísticos descriptivos correspondientes al profesorado

\begin{tabular}{|c|c|c|c|c|}
\hline \multirow{2}{*}{$\begin{array}{l}\text { Descriptivos } \\
\text { Ítems }\end{array}$} & \multicolumn{2}{|c|}{ Pretest } & \multicolumn{2}{|c|}{ Postest } \\
\hline & Media & $\begin{array}{l}\text { Desv. } \\
\text { típ }\end{array}$ & Media & $\begin{array}{l}\text { Desv. } \\
\text { típ }\end{array}$ \\
\hline 6. ¿Cómo dirías que te llevas con tus compañeros? & 1.11 & .323 & 3.56 & .512 \\
\hline 7. ¿Cómo dirías que te llevas con los alumnos y las alumnas del centro? & 1.28 & .575 & 3.56 & .727 \\
\hline 8. ¿Qué visión crees que tienen de ti tus compañeros/as? & 1.50 & .786 & 3.44 & .727 \\
\hline 9. ¿Qué visión crees que tienen de ti tus alumnos/as? & 1.44 & .616 & 3.75 & .447 \\
\hline 10. ¿Crees que hay diferencias entre tus normas de clase y las de otros profesores? & 2.00 & .343 & 2.75 & .577 \\
\hline 11. ¿Cómo es tu relación con las familias de tus alumnos/as? & 2.28 & .752 & 3.19 & .403 \\
\hline $\begin{array}{l}\text { 12.1. ¿En qué medida crees que las familias deben participar en la convivencia } \\
\text { del centro? AMPA. }\end{array}$ & 2.00 & 0 & 1.94 & .250 \\
\hline $\begin{array}{l}\text { 12.2. ¿En qué medida crees que las familias deben participar en la convivencia } \\
\text { del centro? Atención a resultados académicos. }\end{array}$ & 1.83 & .383 & 1.44 & .512 \\
\hline $\begin{array}{l}\text { 12.3. ¿En qué medida crees que las familias deben participar en la convivencia } \\
\text { del centro? Cosas complementarias. }\end{array}$ & 1.89 & .323 & 1.87 & .342 \\
\hline $\begin{array}{l}\text { 12.4. ¿En qué medida crees que las familias deben participar en la convivencia } \\
\text { del centro? Planes de convivencia. }\end{array}$ & 1.94 & .236 & 2.00 & 0 \\
\hline $\begin{array}{l}\text { 12.5. ¿En qué medida crees que las familias deben participar en la convivencia } \\
\text { del centro? Si su hijo va mal. }\end{array}$ & 2.00 & 0 & 1.81 & .403 \\
\hline $\begin{array}{l}\text { 12.6. ¿En qué medida crees que las familias deben participar en la convivencia } \\
\text { del centro? Si son llamados. }\end{array}$ & 1.11 & .323 & 1.56 & .629 \\
\hline $\begin{array}{l}\text { 13.1. ¿Cuánto se repiten los enfrentamientos entre los grupos de alumnos/as } \\
\text { y el profesorado?. }\end{array}$ & 2.00 & .840 & 2.88 & .719 \\
\hline 13.2. ¿Cuánto se repiten las malas contestaciones en clase? & 1.39 & .698 & 3.31 & .946 \\
\hline 13.3. ¿Cuánto se repiten los incumplimientos de normas? & 1.67 & .767 & 2.88 & .806 \\
\hline 13.4. ¿Cuánto se repiten los insultos entre el alumnado? & 1.44 & .616 & 3.31 & .873 \\
\hline 13.5. ¿Cuánto se repiten las peleas entre el alumnado? & 1.44 & .705 & 2.94 & .854 \\
\hline 13.6. ¿Cuánto se repiten los enfrentamientos entre grupitos que no se llevan bien? & 2.39 & .608 & 2.44 & .814 \\
\hline 13.7. ¿Hay niños/as que no están integrados/as y se sienten solos/as? & 2.83 & .514 & 1.94 & .443 \\
\hline 13.8. ¿Los profesores del centro van cada uno a lo suyo? & 1.83 & .786 & 3.50 & .632 \\
\hline $\begin{array}{l}\text { 13.9. ¿Consideras que los/as alumnos/as piensan que los/as profesores/as } \\
\text { no les entienden? }\end{array}$ & 2.61 & .778 & 1.75 & .447 \\
\hline $\begin{array}{l}\text { 13.10. ¿Crees que los/as alumnos/as están desmotivados/as y se aburren } \\
\text { en el centro? }\end{array}$ & 2.00 & .594 & 2.38 & .957 \\
\hline Total & 1.81 & .120 & 2.64 & .256 \\
\hline
\end{tabular}

Como puede observarse en la tabla IV y la figura 4, hay dos tendencias en las respuestas obtenidas: determinados ítems han experimentado una evolución positiva, de la variable 6 a la 11 y de la 12.6 a la 13.5, incluyendo el ítem 13.8, donde los resultados son realmente motivadores y esperanzadores; la actitud y respuestas denotan cambios y perspectivas de seguir apostando por el funcionamiento del programa. 


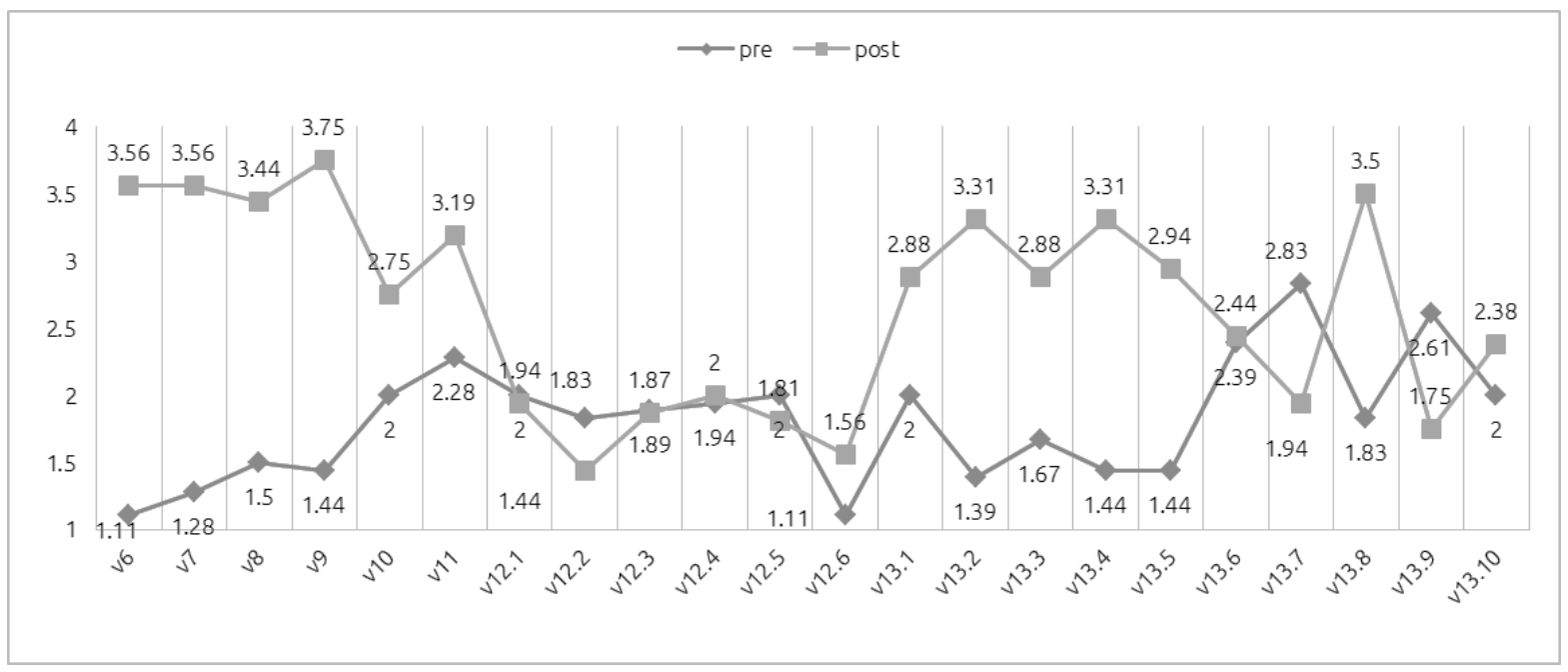

Figura 4. Comparación de medias obtenidas en pretest y postest en el profesorado del centro

Aun así, es necesario recalcar, en primer lugar, los ítems 12.2 y 12.5, correspondientes a la participación de las familias en el centro educativo, lo cual sigue motivando nuestra intención de seguir por esa línea de cara a futuras investigaciones. Respecto al ítem 13.7, referente a la inclusión de determinados alumnos del centro, sin duda la preocupación por el profesorado es evidente. Por el contrario, los resultados obtenidos por el alumnado no corresponden con la misma perspectiva.

Se observa también que el ítem 13.9 (entendimiento entre profesorado y alumnado) coincide plenamente en la percepción del alumnado. Por tanto, aunque no sea positivo es interesante que ambas audiencias transmitan la misma percepción, ya que sólo así se puede seguir avanzando con el objetivo de trabajar en la misma línea.

Por último, en la tabla $V$ se presentan las pruebas realizadas, con la finalidad de conocer la fiabilidad del programa evaluando las diferencias estadísticamente significativas entre ambos momentos de la evaluación y respecto a los tres grupos de estudio.

Tabla V. Relación de diferencias estadísticamente significativas

\begin{tabular}{|c|c|c|c|c|}
\hline \multirow[b]{2}{*}{ Grupo de estudio } & \multicolumn{2}{|c|}{ Prueba Wilcoxon } & \multicolumn{2}{|l|}{ tstudent } \\
\hline & z & $\begin{array}{c}\text { Sig. } \\
\text { Asint. }\end{array}$ & 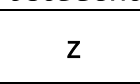 & $\begin{array}{l}\text { Sig. } \\
\text { Asint. }\end{array}$ \\
\hline $\begin{array}{l}\text { Pre y post } \\
\text { Infantil y } 1 \text { er. ciclo }\end{array}$ & -3.966 & 0.000 & -4.611 & 0.000 \\
\hline $\begin{array}{l}\text { Pre y post } \\
\text { 20. y 3er. ciclo }\end{array}$ & -4.560 & 0.000 & -7.354 & 0.000 \\
\hline $\begin{array}{l}\text { Pre y post } \\
\text { Profesorado }\end{array}$ & -3.424 & 0.001 & -10.299 & 0.000 \\
\hline
\end{tabular}

Los resultados obtenidos en ambas pruebas realizadas corroboran la fiabilidad del programa, ya que existen diferencias estadísticamente significativas entre el pretest y el postest en las tres audiencias implicadas.

\section{Discusión y conclusiones}

De acuerdo con el objetivo de la investigación presentada -evaluar la efectividad del programa en materia de convivencia escolar-, después de analizar los resultados antes expuestos, podemos afirmar que la implementación del programa de ayuda entre iguales y mediación artística ha mejorado notablemente la convivencia escolar, tanto desde la perspectiva del profesorado como del alumnado. 
A nivel internacional se han desarrollado diversos estudios en línea como el que se ha presentado, obteniendo resultados que corroboran el adecuado funcionamiento de los mismos. Cowie, Naylor, Talamelli, Chauhan y Smith (2002) analizan los resultados obtenidos después de poner en funcionamiento un programa de formación en sistemas de ayuda entre iguales, donde el alumnado participante afirma que ha contribuido positivamente en el desarrollo de habilidades y estrategias para afrontar dificultades y conflictos en la escuela. Naylor y Cowie (1999) también realizaron una investigación después de la implementación de estrategias de ayuda entre iguales, destacando principalmente la adquisición de habilidades sociales e interpersonales parte del alumnado. Permuy, Méndez y Barreiro (2015), después de la puesta en marcha del programa de alumnos ayudantes, también consideran que se ha mejorado de forma notable la convivencia en el centro en general y en las aulas en particular. Por tanto, podemos afirmar que, una vez más, el desarrollo de este tipo de programas promueve la cultura de paz y la formación de una ciudadanía más democrática, justa y solidaria. Los datos ponen de manifiesto que ambos grupos de estudio sienten que el clima del centro es más positivo y, por tanto, el índice de violencia escolar o conducta disruptiva ha bajado respecto al inicio del estudio.

Aun siendo conscientes de la eficacia del programa, consideramos que las variables destacadas a continuación han influido positivamente en los resultados obtenidos:

- Compromiso del claustro de profesorado del centro en la implementación del programa; iniciativa, esfuerzo y dedicación constante a lo largo de todo el proceso.

- Formación específica del profesorado previa a la puesta en funcionamiento del programa ConVivim, dirigido por profesorado especialista en temáticas de convivencia de la Universidad de Valencia.

- La implementación de las diferentes estrategias que componen el programa se han incluido en la rutina del centro de manera gradual, con la finalidad de evitar cambios drásticos que pudiesen influir negativamente, tanto al alumnado como al profesorado.

- Se han realizado seminarios de trabajo con una profesora experta en convivencia de la Universidad de Valencia a lo largo de todo el curso, con la finalidad de asesorar al profesorado que conforma el Observatorio de la Convivencia del centro del funcionamiento de las diferentes estrategias propuestas.

Sin duda, las variables expuestas han potenciado el adecuado funcionamiento del programa, aportando la energía y el profesionalismo necesarios para promover el cambio deseado, centrándose principalmente en la mejora del clima del centro y en la disminución del índice de violencia escolar. Tal y como hemos comprobado en estudios similares (Akguna y Araz, 2014; Fisher y Kettl, 2003; Muñoz, Lucero, Cornejo, Muñoz y Araya, 2014), disminuir la agresividad entre el alumnado es un camino largo de recorrer y sólo se consigue con dedicación y esfuerzo por parte de la comunidad educativa, apostando por una escuela inclusiva. Los efectos de la implementación de este tipo de programas de mejora de la convivencia escolar resultan evidentes a largo plazo, por lo que incidimos en la importancia de seguir trabajando en su desarrollo en cursos posteriores, con el objetivo de que se convierta en parte de la identidad del centro.

Aun así, resulta fundamental realizar una reflexión en torno a la mejora del programa implementado. Destacamos, en primer lugar, la necesidad de potenciar y promover la participación de las familias en el centro, ya que somos conscientes de la importancia que tiene que padres, madres y familia extensa formen parte activa de la comunidad educativa (Páez, 2015). No cabe duda que investigadores reconocidos en el ámbito educativo han corroborado los efectos positivos de ello (Flecha, 2015).

Por otra parte, es importante mencionar la exigencia de construir una escuela que abogue por convertirse en una institución abierta al barrio y a la comunidad. Sentirse parte de algo, siempre implica valorarlo y amarlo y es por ello por lo que consideramos que si los ciudadanos del barrio en el que está situada la escuela consideran que es un lugar del que ellos también forman parte, conseguiremos que la respeten, la valoren y la amen. 
A raíz de esta reflexión, como futuras líneas de investigación, estamos trabajando para construir un proyecto de escuela como centro e institución referente en el barrio, con el objetivo de abrirla a la participación de la comunidad. Para ello, la implicación tanto de familias como del barrio es fundamental. El primer paso es abrir las puertas de la escuela en horario extraescolar, ofreciendo sus espacios de cara a la realización de talleres y actividades formativas, dirigidas a toda la comunidad, actividades que serán dinamizadas por estudiantes de las titulaciones de Educación Social y Pedagogía de la Universidad de Valencia, con la finalidad de unir instituciones que nunca deberían haberse alejado. Y es que "mucha gente pequeña, en lugares pequeños, haciendo cosas pequeñas, puede cambiar el mundo", en palabras del recordado Eduardo Galeano.

Como docentes estamos preocupados porque nuestros estudiantes comprendan. Según Ontoria (2003, p. 21), "cuando se habla de que los alumnos comprendan, estamos diciendo que intenten dar sentido a aquello con lo que entran en contacto y mediante lo cual se forman las representaciones y los esquemas cognitivos", por tanto, en nuestras clases los estudiantes deben dar sentido a los diferentes conceptos que se trabajan y formar esquemas cognitivos, los cuales se ven reflejados en la construcción de mapas conceptuales. Así, algunas de las tareas que podrían incluirse dentro de nuestros cursos de formación de profesores es el desarrollo de mapas conceptuales, tanto sobre el conocimiento disciplinar como el pedagógico.

\section{Referencias}

Akguna, S. y Araz, A. (2014). The effects of conflict resolution education on conflict resolution skills, social competence, and aggression in Turkish elementary school students. Journal of Peace Education, 11(1), 3045. doi:10.1080/17400200903370928

Boqué, M., Pañellas, M., Alguacil, M. y García, L. (2014). La cultura de paz en la educación para la ciudadanía y los derechos humanos en los libros de texto de educación primaria. Perfiles Educativos, 36(146), 80-97.

Caba-Collado, M. A. y López-Atxurra, R. (2013). La competencia de ayuda entre iguales para evitar la agresión en la escuela. Revista Internacional de Educación para la Justicia Social, 2(1), 119-138.

Cangas, A., Gázquez, J., Pérez-Fuentes, M., Padilla, D. y Miras, F. (2007). Evaluación de la violencia escolar y su afectación personal en una muestra de estudiantes europeos. Psicothema, 19(1), 114-119.

Cowie, H. y Fernández, F. (2006). Ayuda entre iguales en las escuelas: desarrollo y retos. Revista Electrónica de Investigación Socioeducativa, 4(2), 291-310.

Cowie, H., Naylor, P., Talamelli, L., Chauhan, P. y Smith, P. (2002). Knowledge, use of and attitudes towards peer support. Journal of Adolescence, 25(2), 453-467. doi: 10.1006/jado.2002.0498

Cowie, H. y Wallace, P. (2000). Peer support in action. Londres: Sage.

Durán, D. y Blanch, S. (2015). Read one: un programa de mejora de la lectura a través de la tutoría entre alumnos y el apoyo familiar. Cultura y Educación, 19(1), 31-45. doi:10.1174/113564007780191287

Fernández, I. (2008). Los programas de ayuda para la mejora de la convivencia en instituciones educativas. Bordón. Revista de pedagogía, 60(4), 137-150.

Fisher, K. y Kettl, P. (2003). Teachers' perceptions of school violence. Journal of Pediatric Health Care, 17(2), 79-83.

Flecha, J. (2015). Comunidades de aprendizaje: sueños posibles para todos los niños y las niñas. Aula de Innovación Educativa, 241, 12-16. 
Freinet, C. (1971). Las invariantes pedagógicas. Barcelona: Estela.

Funes, S. (2000). Resolución de conflictos en la escuela: Una herramienta para la cultura de paz y la convivencia. Contextos Educativos, 3, 91-106.

Gázquez, J., Cangas, A., Pérez, M. y Lucas, F. (2009). Teachers' perception of school violence in a sample from three european countries. European Journal of Psychology of Education, 24(1), 49-59.

Gómez, S. y Gaymard, S. (2014). The perception of school climate in two secondary schools during the implementation of a peer support program. Electronic Journal of Research in Educational Psychology, 12(2), 509-540.

Grau, R., García-Raga, L. y López-Martín, R. (2016). Towards school transformation. Evaluation of a coexistence program from the voice of students and teachers. Journal of New Approaches in Educational Research, 5(2), 137-146. doi:10.7821/паег.2016.7.177

Harber, C. y Sakade, N. (2009). Schooling for violence and peace: how does peace education differ from 'normal' schooling? Journal of Peace Education 6(2), 171-187. doi:10.1080/17400200903086599

Moreno, A. (2010). La mediación artística: un modelo de educación artística para la intervención social a través del arte. Revista Iberoamericana de Educación, 52(2), 1-9.

Muñoz, M., Lucero, B., Cornejo, C. Muñoz, P. y Araya, N. (2014). Convivencia y clima escolar en una comunidad educativa inclusiva de la Provincia de Talca, Chile. Revista Electrónica de Investigación Educativa, 16(2), 16-32. Recuperado de https://redie.uabc.mx/redie/article/view/497

Naylor, P. y Cowie, H. (1999). The effectiveness of peer support systems in challenging school bullying: the perspectives and experiences of teachers and pupils. Journal of Adolescence, 22(4), 467-479. doi:10.1006/jado.1999.0241.

Ortega, R. y Del Rey, R. (2003). La violencia escolar. Estrategias de prevención. Barcelona: Graó.

Páez, R. (2015). Familia y escuela: Una alianza necesaria en la gestión escolar. Revista Iberoamericana de Educación, 67, 159-180.

Permuy, A., Méndez, M. J. y Barreiro, F. (2015). O alumnado axudante como figura chave para construción dunha convivencia positiva [Estudiantes asistentes como figuras clave para construir una coexistencia positiva]. Revista de Estudios e Investigación en Psicología y Educación, 2(Ext.), 57-59.

Puig, M. y Morales, J. (2015). La formación de ciudadanos: conceptualización y desarrollo de la competencia social y cívica. Educación XXI, 18(1), 259-282. doi:10.5944/educXX1.18.1.12332

Rodrigo-Montero, J. (2015). Kunstcoop: Experiencias de mediación artística en Alemania. Arte, individuo y sociedad, 27(3), 373-392. doi:10.5209/rev-ARIS.2015.v27.n3.4372

Santos-Guerra, M. A. (Coord.) (2003). Aprender a convivir en la escuela. Madrid: Akal.

Santos-Guerra, M. A. (2009). La escuela que aprende. Retos, dificultades y esperanzas. En M. A. SantosGuerra (Ed.), Escuelas para la democracia. Cultura, organización y dirección de instituciones educativas (pp. 163-184). Madrid: Wolters Kluwer.

Schulman, M. (2002). How we become moral. En C. R. Snyder y López, S. J. (Eds.), The Oxford handbook of positive psychology (499-512). Oxford University Press.

Torrego, J. (2013). La ayuda entre iguales para la mejora de la convivencia escolar. Madrid: Narcea. 
UNESCO. (2015). Rethinking education. Towards a global common good? Francia: Autor.

Viguer, P. y Avià, S. (2009). Un modelo local para la promoción de la convivencia y la prevención de la violencia entre iguales desde el ámbito comunitario. Cultura y Educación, 21(3), 345-359.

Yubero, S., Larrañaga, E. y Morales, J. (2003). La sociedad educadora. Cuenca: Colección Estudios.

Valdebenito, V. y Duran, D. (2015). The coordinating role of the teacher in a peer tutoring programme. Procedia. Social and Behavioral Sciences, 191, 2300-2306. doi:10.1016/j.sbspro.2015.04.423

Volk, A., Dane, A., Marini, Z. y Vallancourt, T. (2015). Adolescent bullying, dating, and mating: testing an evolutionary hypothesis. Evolutionary Psychology, 13(4), 1-11. doi:10.1177/1474704915613909 